\title{
Prospectiva estratégica al sector textil del distrito de San Juan de Lurigancho
}

\section{RESUMEN}

El presente artículo trata el siguiente problema ¿cómo formular un escenario estratégico para el sector textil del distrito de San Juan de Lurigancho?, para lograr eso se utilizó el método francés de Michael Godet de la prospectiva estratégica, y así se obtuvo una serie de escenarios prospectivos posibles seleccionando de entre ellos el escenario apuesta, identificando previamente las variables principales y el comportamiento de los actores en el sistema.

Palabras clave: Prospectiva, Escenarios, Sector Textil, Estratégico

STRATEGIC PROSPECTIVE TO THE TEXTILE SECTOR OF THE District of San Juan LuRIGancho

\section{ABSTRACT}

This article is about the next problem inow to formulate a strategic scenario to textile sector of San Juan de Lurigancho district?, in order to achieved the French Method of the Strategic Prospective was used, being obtained a series of possible prospective scenarios, selecting among them the bets scenario, identifying previously the principals variables and the system actors behavior.

Keywords: Prospective, Scenarios, Textile Area, Strategic

\section{INTRODUCCIÓN}

El estudio del futuro es un tema bastante recurrente no sólo en líderes de organizaciones sino también en el común de las personas. Dentro de esta premisa la prospectiva del sector textil, es la línea de base para programas y planes estratégicos sectoriales, en especial, en la región Lima y el distrito de San Juan de Lurigancho. La industria textil y de confecciones es la fuente más importante de puestos de trabajo en el sector industrial peruano.

Para tener una visión general del escenario actual, según el Ministerio de Comercio Exterior y Turismo (MINCETUR) aproximadamente el $10 \%$ de toda la pobl ación peruana depende de la industria textil. Cada puesto de trabajo directo genera dos puestos indirectos en toda la economía, debido a la alta concentración de pequeñas empresas del sector textil, especialmente en San Juan de Lurigancho, quienes abastecen al conglomerado de Gamarra, asimismo, es el sector que genera más empleo directo.

El estudio contribuye con el sector textil de San Juan de Lurigancho, en el sentido de que orientará el rumbo y las tendencias tecnológicas de dicho sector hacia un escenario que considere todos los anhelos y deseos de los actores involucrados.

Por otro lado, se presentará una metodología que se podrá aplicar a sectores similares, siempre y cuando se tomen en consideración las diferencias existentes, esto en el marco del método francés propuesto por el laboratorio LIPSOR y fundamentado por Michael Godet; bajo la premisa de que el futuro no se adivina sino se construye. Este método permite tomar en consideración datos cualitativos y evaluarlos en forma cuantitativa mediante la construcción de diversas matrices.

El tema es inédito para San Juan de Lurigancho. Hasta la fecha, no se ha efectuado un estudio textil en dicha zona, sin embargo, se puede mencionar como antecedente directo el proyecto Prospectiva Estratégica del Cluster de Muebles de Villa El Salvador (Lima-Perú), estudio desarrollado por el mismo equipo de investigación prospectiva de la Facultad de Ingeniería Industrial

1 Magíster en ciencias. Profesor del Departamento de Diseño y Tecnología Industrial. UNMSM. E-mail: jlinche@hotmail.com

2. Magíster en Ingeniería Industrial. Profesor del Departamento de Diseño y Tecnología Industrial. UNMSM. E-mail: ramon_chung@yahoo.es

3. Ingeniero Industrial. Profesor del Departamento de Diseño y Tecnología Industrial. UNMSM. E-mail: c.c.cesar@yahoo.es 
de la UNMSM, artículo publicado en la revista Industrial Data Vol. $9 \mathrm{~N}^{\circ} 1$.

La formulación consta de tres partes:

1. Análisis MIC MAC
2. Análisis MACTOR
3. Análisis SMIC

A continuación se explican los resultados.

\section{ANÁLISIS MIC MAC}

Luego de la consulta a expertos (gremios textiles, empresarios, maestristas, etc.) se identificaron 26 variables de gran importancia para el sector en estudio las cuales se listan en el Cuadro 1.

Estas 26 variables posteriormente son cruzadas en una matriz de impactos directos, en donde cada celda representa la relación de dos variables; luego se le asigna un valor, el cual representa la intensidad de dicha relación, el valor sigue el siguiente criterio:
0 : No tiene influencia

1: Influencia débil

2: Influencia media

3: Influencia fuerte

$\mathrm{P}$ : Influencia potencial

Los resultados se muestran en el Cuadro 2; posteriormente y elevando esta matriz a sucesivas potencias $(1,2,3 \ldots)$ hasta que sea estable, es decir que el orden de los factores no se altere, se logró la Matriz de Impactos Indirectos mostrada en el Cuadro 3; luego en dicha matriz, la sumatoria de cada columna proporciona el valor de dependencia y la sumatoria de los valores de cada fila el valor de dependencia todo esto por cada variable.

A continuación se analiza la naturaleza de las variables, para esto se coloca en el eje $Y$ el valor de influencia y en el eje $X$ el de dependencia, obteniéndose la Figura 1. Es necesario poner bastante atención en las variables claves, ya que estas tienen una gran influencia pero también una gran dependencia, por lo tanto, son inestables pero con capacidad de alterar todo el sistema estudiado, el Cuadro 4 presenta dichas variables.

\section{CUADRO 1: VARIABLES IDENTIFICADAS}

\begin{tabular}{|c|c|c|}
\hline $\mathbf{N}^{\circ}$ & Título LARGo & TÍtULO CORTO \\
\hline 1 & Capacitación & VAR1 \\
\hline 2 & Relaciones cliente proveedor & VAR2 \\
\hline 3 & Integración vertical & VAR3 \\
\hline 4 & Aprovechamiento de oportunidades & VAR4 \\
\hline 5 & Ubicación & VAR5 \\
\hline 6 & Seguridad del distrito & VAR6 \\
\hline 7 & Optimización de costos & VAR7 \\
\hline 8 & Demanda de exportación & VAR8 \\
\hline 9 & Facilidades para la constitución de empresas & VAR9 \\
\hline 10 & FINANCIAMIENTO & VAR10 \\
\hline 11 & Difusión hacia empresarios textiles para invertir en empresas en el distrito & VAR11 \\
\hline 12 & Capacidad de producción & VAR12 \\
\hline 13 & Calidad de los centros de formación textil & VAR13 \\
\hline 14 & Disponibilidad de negocios afines y complementarios al sector textil & VAR14 \\
\hline 15 & Nivel de márgenes de ganancia & VAR15 \\
\hline 16 & Generación de empleo dependiente en el rubro & VAR16 \\
\hline 17 & Generación de empresas familiares & VAR17 \\
\hline 18 & Calidad de las decisiones del Estado en el sector textil & VAR18 \\
\hline 19 & Oferta exportable de prendas al mercado europeo & VAR19 \\
\hline 20 & Marco legal sobre el control y supervisión de residuos sólidos & VAR20 \\
\hline 21 & Infraestructura y maquinaria para la producción textil & VAR21 \\
\hline 22 & Sustitución de insumos textiles & VAR22 \\
\hline 23 & Nivel de competencia & VAR23 \\
\hline 24 & Ética en las negociaciones de importación y exportación & VAR24 \\
\hline 25 & Formalización de las empresas del sector & VAR25 \\
\hline 26 & Apertura a la importación de productos textiles a precios bajos & VAR26 \\
\hline
\end{tabular}

Elaboración del grupo de trabajo. 


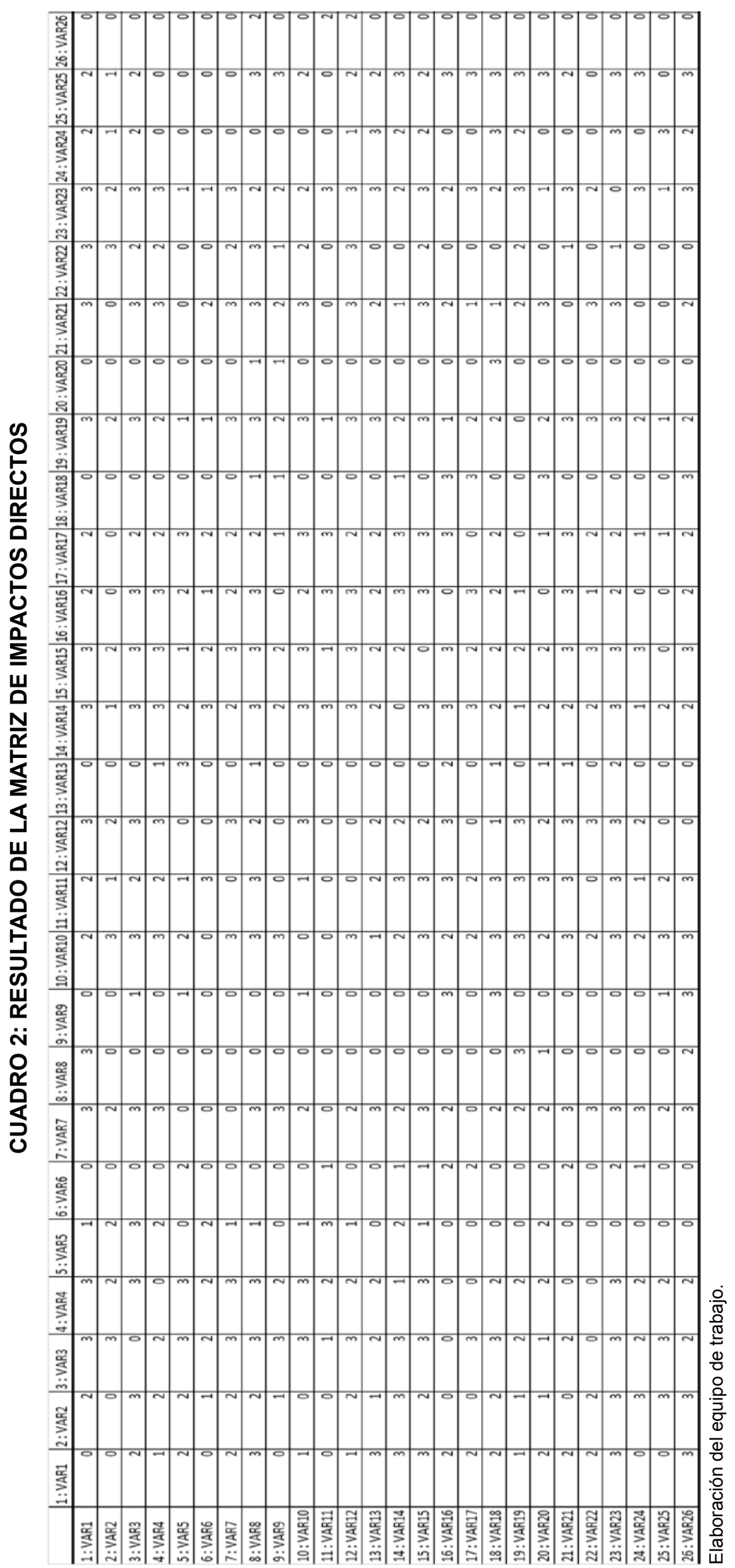




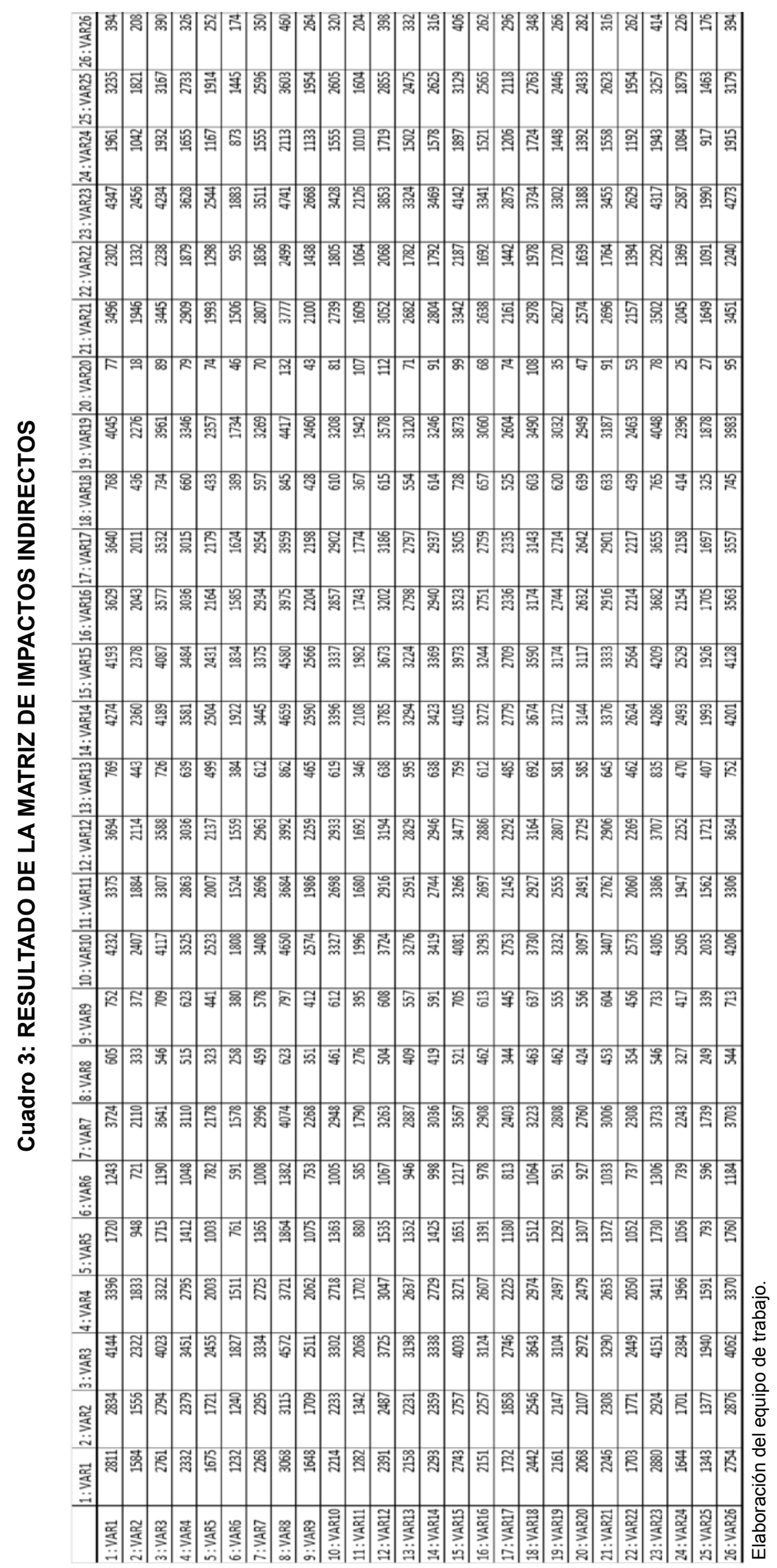


FIGURA 1: UBICACIÓN DE LAS VARIABLES EN EL PLANO

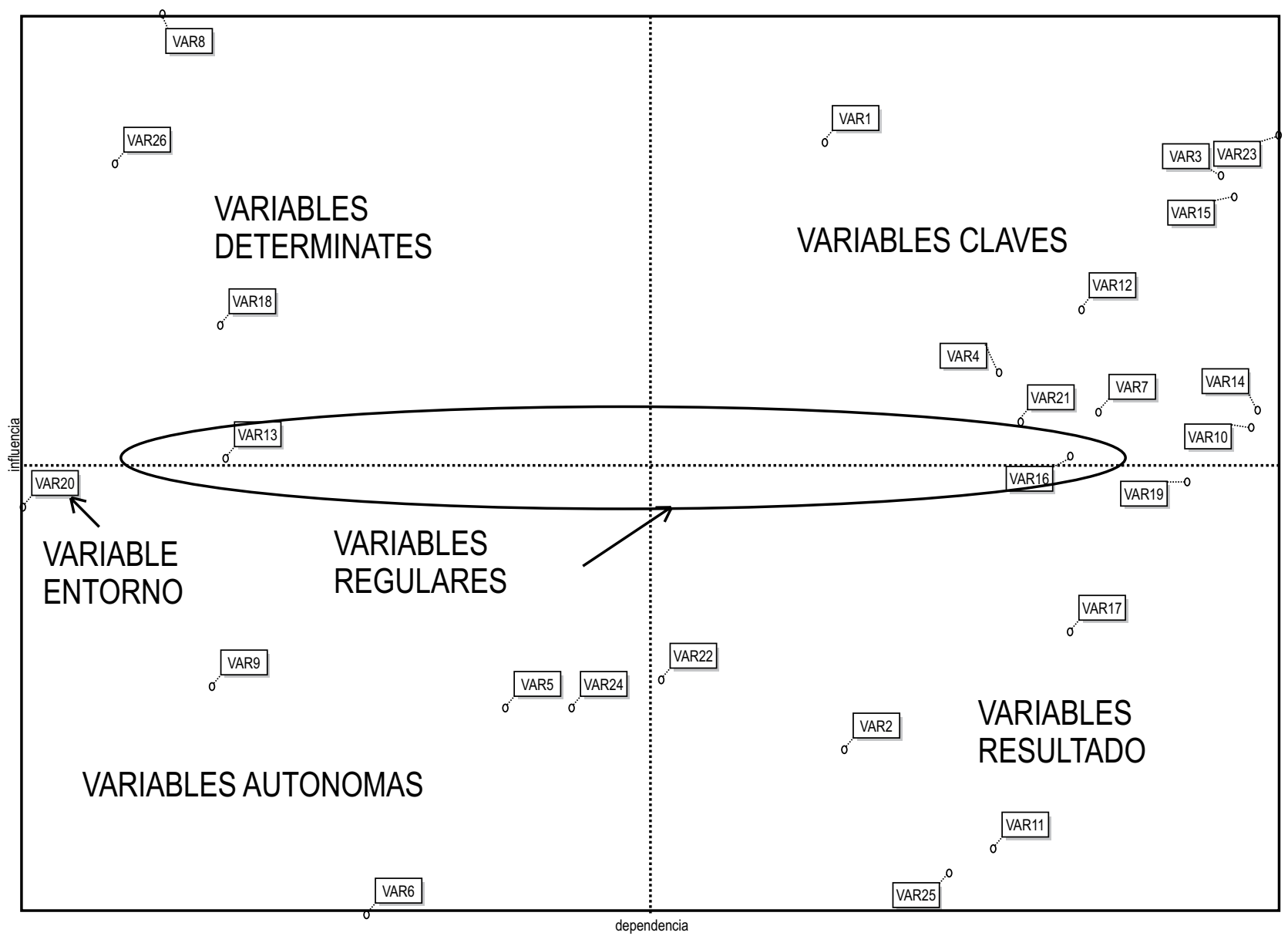

CUADRO 4: VARIABLES CLAVE

\begin{tabular}{|l|l|}
\hline Título largo & Título corto \\
\hline Capacitación & VAR1 \\
\hline Integración vertical & VAR3 \\
\hline Aprovechamiento de Oportunidades & VAR4 \\
\hline Optimización de costos & VAR7 \\
\hline Financiamiento & VAR10 \\
\hline Capacidad de producción & VAR12 \\
\hline Disponibilidad de negocios afines y complementarios al sector textil & VAR14 \\
\hline Nivel de márgenes de ganancia & VAR15 \\
\hline Infraestructura y maquinaria para la producción textil & VAR21 \\
\hline Nivel de competencia & VAR23 \\
\hline
\end{tabular}

Fuente: Elaboración del grupo de trabajo.

\section{RESULTADOS DEL ANÁLISIS MACTOR}

Para la realización de este análisis se identificaron, junto con los expertos, los principales actores del sistema en estudio cuyos resultados se presentan en el Cuadro 5.
Por otro lado y en diferentes sesiones de trabajo, se identificaron los principales objetivos del sistema estudiado, cuya relación se presenta en el Cuadro 6. 
CUADRO 5: LISTA DE ACTORES IDENTIFICADOS

\begin{tabular}{|l|l|l|}
\hline$N^{0}$ & Título largo & Título corto \\
\hline 1 & PROMPERU & ACT1 \\
\hline 2 & Empresarios textiles del distrito & ACT2 \\
\hline 3 & Ministerio de la Producción & ACT3 \\
\hline 4 & Asociaciones y gremios del sector en el distrito & ACT4 \\
\hline 5 & Ministerio del Ambiente & ACT5 \\
\hline 6 & Municipalidad del distrito & ACT6 \\
\hline 7 & Gobierno Regional de Lima & ACT7 \\
\hline 8 & Ministerio de trabajo & ACT8 \\
\hline 9 & ONG's & ACT9 \\
\hline 10 & Universidades & ACT10 \\
\hline 11 & Institutos tecnológicos textiles & ACT11 \\
\hline 12 & Clientes & ACT12 \\
\hline 13 & Bancos y financieras & ACT13 \\
\hline 14 & Empleados dependientes del sector & ACT14 \\
\hline
\end{tabular}

CUADRO 6: RELACIÓN DE OBJETIVOS IDENTIFICADOS

\begin{tabular}{|c|l|l|}
\hline $\mathrm{N}^{\circ}$ & Título largo & Título corto \\
\hline 1 & Implementar tratados de libre comercio con la UE & OBJ1 \\
\hline 2 & Incrementar la producción de prendas de vestir con algodón orgánico & OBJ2 \\
\hline 3 & Implementar sistemas de gestión integrada de la calidad & OBJ3 \\
\hline 4 & Implementar de sistemas de costos adecuados & OBJ4 \\
\hline 5 & Participar en ferias nacionales e internacionales & OBJ5 \\
\hline 6 & Exportar los productos textiles & OBJ6 \\
\hline 7 & Incrementar la capacitación del personal & OBJ7 \\
\hline 8 & Establecer redes de colaboración para la venta de productos textiles & OBJ8 \\
\hline 9 & Implementar criterios y herramientas adecuados de gestión & OBJ9 \\
\hline 10 & Liderar el sector textil & OBJ10 \\
\hline 11 & Fortalecer las actividades relacionadas al sector textil & OBJ11 \\
\hline 12 & Implementar mecanismos para la constitución de empresas en el menor tiempo posible & OBJ12 \\
\hline 13 & Fomentar la creación de nuevos centros superior en el sector textil en SJL & OBJ13 \\
\hline 14 & Facilitar mecanismos financieros para nuevos proyectos relacionados al sector en SJL & OBJ14 \\
\hline 15 & Mejorar la reputacion e imagen del sector en SJL & OBJ15 \\
\hline 16 & Mejorar los empaques y etiquetas del producto final & OBJ16 \\
\hline 17 & Mejorar y optimizar el aspecto y tamaño de las instalaciones & OBJ17 \\
\hline 18 & Optimizar el lead time de entrega de la sempresas en el sector & OBJ18 \\
\hline 19 & Aumentar la participación en el mercado del sector & OBJ19 \\
\hline
\end{tabular}

Fuente: Elaboración del grupo de trabajo.

Con todos estos datos se procedió a aplicar la matriz directa de actores $\mathrm{x}$ actores, sin embargo, similar al caso MIC MAC, esta matriz sólo toma en cuenta las relaciones directas, por lo tanto se procedió a formular la matriz indirecta cuyo resultado se puede apreciar en el Cuadro 7. Los cálculos son similares a los hechos en el análisis MIC MAC, con la salvedad de que en el grado de relación entre actores se consideró lo siguiente:
4: el actor Ai puede cuestionar la existencia del actor Aj.

3: el actor Ai puede cuestionar las misiones del actor Aj.

2: el actor Ai puede cuestionar los proyectos del actor Aj.

1: el actor Ai puede cuestionar, de manera limitada (durante algún tiempo o en algún caso concreto) la operativa del actor $\mathrm{Aj}$. 
CUADRO 7: MATRIZ DE IMPACTO INDIRECTO

\begin{tabular}{|l|r|r|r|r|r|r|r|r|r|r|r|r|r|r|r|}
\hline & ACT1 & ACT2 & ACT3 & ACT4 & ACT5 & ACT6 & ACT7 & ACT8 & ACT9 & ACT10 & ACT11 & ACT12 & ACT13 & ACT14 & li \\
\hline ACT1 & 10 & 11 & 10 & 11 & 2 & 7 & 4 & 4 & 9 & 8 & 7 & 4 & 4 & 4 & 85 \\
\hline ACT2 & 18 & 18 & 14 & 21 & 6 & 8 & 8 & 9 & 18 & 11 & 15 & 12 & 8 & 12 & 160 \\
\hline ACT3 & 10 & 9 & 6 & 10 & 2 & 8 & 4 & 4 & 8 & 8 & 7 & 4 & 4 & 4 & 82 \\
\hline ACT4 & 20 & 20 & 16 & 21 & 6 & 10 & 10 & 9 & 19 & 13 & 15 & 12 & 8 & 12 & 170 \\
\hline ACT5 & 18 & 20 & 14 & 21 & 4 & 13 & 12 & 6 & 18 & 14 & 15 & 8 & 8 & 8 & 175 \\
\hline ACT6 & 15 & 16 & 13 & 19 & 4 & 11 & 10 & 4 & 19 & 12 & 15 & 9 & 7 & 9 & 152 \\
\hline ACT7 & 15 & 17 & 12 & 21 & 4 & 11 & 9 & 6 & 17 & 12 & 13 & 10 & 5 & 10 & 153 \\
\hline ACT8 & 14 & 16 & 10 & 19 & 4 & 12 & 10 & 4 & 18 & 10 & 13 & 10 & 6 & 10 & 152 \\
\hline ACT9 & 16 & 16 & 12 & 16 & 2 & 11 & 8 & 4 & 14 & 14 & 13 & 4 & 10 & 4 & 130 \\
\hline ACT10 & 13 & 13 & 9 & 14 & 2 & 10 & 8 & 2 & 14 & 11 & 12 & 4 & 10 & 4 & 115 \\
\hline ACT11 & 4 & 4 & 4 & 4 & 2 & 4 & 2 & 2 & 4 & 2 & 2 & 2 & 1 & 2 & 37 \\
\hline ACT12 & 18 & 19 & 18 & 21 & 4 & 10 & 8 & 7 & 20 & 13 & 17 & 14 & 8 & 14 & 177 \\
\hline ACT13 & 23 & 23 & 19 & 26 & 6 & 11 & 10 & 9 & 23 & 15 & 20 & 15 & 10 & 15 & 215 \\
\hline ACT14 & 20 & 21 & 16 & 23 & 6 & 12 & 8 & 9 & 19 & 13 & 15 & 10 & 8 & 10 & 180 \\
\hline Di & 204 & 205 & 167 & 226 & 50 & 127 & 102 & 75 & 206 & 145 & 177 & 104 & 87 & 108 & 1983 \\
\hline
\end{tabular}

Fuente: Elaboración del grupo de trabajo.

\section{FIGURA 2: PLANO DE INFLUENCIA DEPENDENCIA DE ACTORES}

Plano de influencias y dependencias entre actores

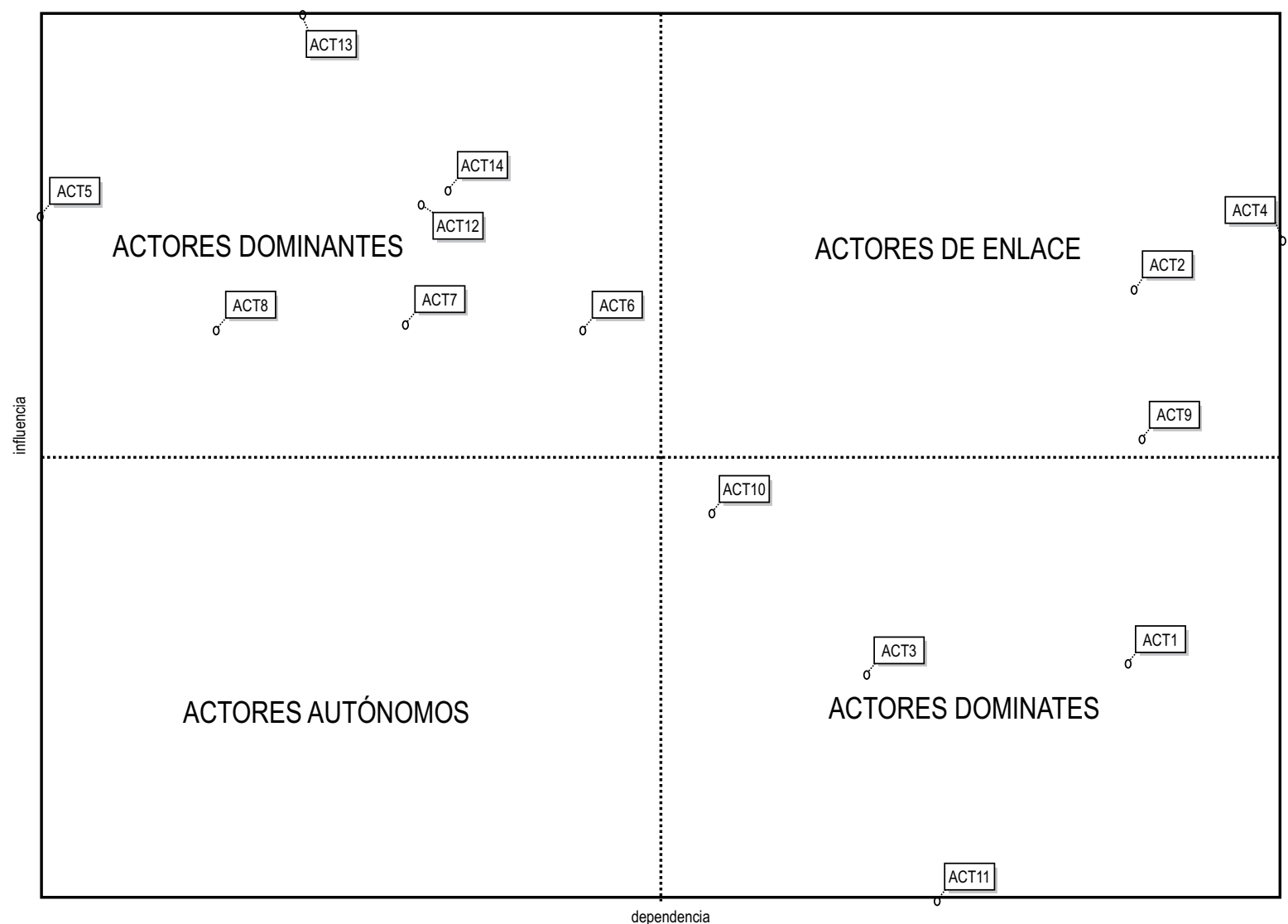

0: el actor Ai no tiene ninguna influencia sobre el actor $\mathrm{Aj}$.

Asimismo, como en el caso de los factores, es necesario conocer el comportamiento de estos actores a fin de poder formular las estrategias para llegar a ellos y lograr su apoyo o neutralizarlos para evitar o disminuir su ataque; la Figura 2 muestra el plano de influencia y dependencia de los actores e identifica a cada actor según su posición. 


\section{CUADRO 8: HIPÓTESIS PARA LA FORMULACIÓN DE LOS ESCENARIOS}

\begin{tabular}{|c|c|c|}
\hline $\mathbf{N}^{\circ}$ & TÍtULO LARGo & ESCENARIO HIPOTÉTICO \\
\hline 1 & Capacitación & $\begin{array}{l}\text { En el año } 2025 \text { los empresarios del sector estarán } \\
\text { debidamente actualizados en herramientas de gestión } \\
\text { y en exportaciones, propiciando la generación de } \\
\text { negocios internacionales sostenibles (es decir, pedidos } \\
\text { constantes por cliente con duración de un año como } \\
\text { mínimo), asimismo, habrán cambiado su enfoque } \\
\text { empresarial actual a uno duradero en el tiempo. } \\
\text { Por su parte los trabajadores también estarán } \\
\text { capacitados en el manejo de sus equipos, así como en } \\
\text { la solución de problemas de planta mediante círculos } \\
\text { de calidad entre otras herramientas. } \\
\text { En todos los casos, la capacitación será permanente y } \\
\text { periódica. }\end{array}$ \\
\hline 2 & Integración vertical & $\begin{array}{l}\text { Las empresas habrán logrado una integración con sus } \\
\text { proveedores, a quienes los considerarán como parte } \\
\text { de la cadena de valor y en muchos casos estarán } \\
\text { integrados a sus organizaciones. } \\
\text { Entre las herramientas de integración se contará } \\
\text { con las relacionadas a contratos permanentes } \\
\text { de abastecimiento generados por exportaciones } \\
\text { constantes. }\end{array}$ \\
\hline 3 & Aprovechamiento de oportunidades & $\begin{array}{l}\text { Las empresas contarán con un área encargada de } \\
\text { la detección de nuevas oportunidades en diversos } \\
\text { campos (negocios, logístico, etc.), lo que permitirá a } \\
\text { estos empresarios aprovechar y desarrollar su oferta } \\
\text { tanto nacional como internacional. }\end{array}$ \\
\hline 4 & Optimización de costos & $\begin{array}{l}\text { En cuanto a costos, las empresas habrán superado } \\
\text { los conceptos actuales de costos y trabajarán con } \\
\text { estructuras de costos adecuadas, las cuales le } \\
\text { proporcionarán información real y oportuna (contarán } \\
\text { con conexión en línea para dicha información), con lo } \\
\text { cual mejorarán las utilidades de dicha empresas. }\end{array}$ \\
\hline 5 & Financiamiento & $\begin{array}{l}\text { Debido a la expansión que experimentarán } \\
\text { las empresas del sector, los requerimientos de } \\
\text { financiamientos para nuevos proyectos o exportaciones } \\
\text { también crecerán en forma significativa. Ello } \\
\text { demostrará que el sector está en crecimiento y genera } \\
\text { bienestar al distrito y a los empresarios y trabajadores. }\end{array}$ \\
\hline
\end{tabular}

Elaboración del grupo de trabajo.

\section{ANÁLISIS SMIC}

Para la formulación de los escenarios se consideraron 5 variables, es decir, las de mayor puntaje y mediante las opiniones de los expertos se formuló una hipótesis por cada variable cuyos resultados se muestran en el Cuadro 8.

Luego del análisis probabilístico se pueden construir las diversas combinaciones de realización o no de las hipótesis, lo cual generará múltiples es- cenarios futuribles ${ }^{1}$ con diversas probabilidades de realización; para el caso del estudio el número 1 significa la realización de la hipótesis y 0 lo contrario, por ejemplo si el escenario 9 se presenta como 9 - 1011, se interpreta como que en el escenario 9 cumplen hipótesis 1,3 y 4 (ya que tienen el valor de 1) pero la hipótesis 2 no se realiza, en la formulación es su negación (ya que tiene valor 0 ). El cuadro 9 muestra dichos resultados.

1 Según el diccionario de la Real Académica Española, futurible se define como: "Se dice de lo futuro condicionado, que no será con seguridad, sino que sería si se diese una condición determinada" 


\section{CUADRO 9: PROBABILIDADES DE ESCENARIOS ORDENADO}

\begin{tabular}{|c|c|c|c|}
\hline ESCENARIO & PROB. INDIV. & PROB. \% & ACUM. (\%) \\
\hline $32-00000$ & 0.263 & 26.3 & 26.3 \\
\hline $01-11111$ & 0.200 & 20.0 & 46.3 \\
\hline $09-10111$ & 0.142 & 14.2 & 60.5 \\
\hline $16-10000$ & 0.073 & 7.3 & 67.8 \\
\hline $30-00010$ & 0.063 & 6.3 & 74.1 \\
\hline $03-11101$ & 0.038 & 3.8 & 77.9 \\
\hline $17-01111$ & 0.029 & 2.9 & 80.8 \\
\hline $28-00100$ & 0.028 & 2.8 & 83.6 \\
\hline $11-10101$ & 0.025 & 2.5 & 86.1 \\
\hline $08-11000$ & 0.024 & 2.4 & 88.5 \\
\hline $04-11100$ & 0.023 & 2.3 & 90.8 \\
\hline $12-10100$ & 0.022 & 2.2 & 93.0 \\
\hline $05-11011$ & 0.019 & 1.9 & 94.9 \\
\hline $13-10011$ & 0.015 & 1.5 & 96.4 \\
\hline $14-10010$ & 0.012 & 1.2 & 97.6 \\
\hline $22-01010$ & 0.012 & 1.2 & 98.8 \\
\hline $25-00111$ & 0.004 & 0.4 & 99.2 \\
\hline $06-11010$ & 0.003 & 0.3 & 99.5 \\
\hline $20-01100$ & 0.003 & 0.3 & 99.8 \\
\hline $18-01110$ & 0.002 & 0.2 & 100.0 \\
\hline $02-11110$ & 0.000 & 0.0 & 100.0 \\
\hline $07-11001$ & 0.000 & 0.0 & 100.0 \\
\hline $10-10110$ & 0.000 & 0.0 & 100.0 \\
\hline $15-10001$ & 0.000 & 0.0 & 100.0 \\
\hline $19-01101$ & 0.000 & 0.0 & 100.0 \\
\hline $21-01011$ & 0.000 & 0.0 & 100.0 \\
\hline $23-01001$ & 0.000 & 0.0 & 100.0 \\
\hline $24-01000$ & 0.000 & 0.0 & 100.0 \\
\hline $26-00101$ & 0.000 & 0.0 & 100.0 \\
\hline $27-00101$ & 0.000 & 0.0 & 100.0 \\
\hline $29-00011$ & 0.000 & 0.0 & 100.0 \\
\hline $31-00001$ & 0.000 & 0.0 & 100.0 \\
\hline
\end{tabular}

Analizando el Cuadro 9, el cual indica la probabilidad de la realización de los posibles escenarios que responden a la combinación de la ocurrencia o no de las hipótesis, se puede apreciar en la columna del acumulado que el núcleo tendencial está dado por 4 escenarios que representan cerca del $68 \%$ de la realización de todas las hipótesis, 3 de ellos tiene una mayor presencia, es decir, el escenario 32,1 y 9; el escenario 16 que solo representa $7 \%$ puede ser descartado del análisis. La probabilidad individual del escenario (columna 2) se calcula mediante minimización cuadrática con restricciones y posteriormente con la aplicación del teorema de Bayes.

\section{SELECCIÓN DEL ESCENARIO APUESTA}

Conforme se desprende del análisis anterior, el escenario denominado "Exterminio total" (Escenario 32-0000) es el que tiene una mayor probabilidad de ocurrencia, lo cual llama la atención porque de seguir las mismas relaciones entre los factores y actores, es decir, que no se haga nada al respecto, es muy probable que ocurra dicho escenario futurible, sin embargo, el escenario "Jardín del Edén" (Escenario 01-1111) también tiene una fuerte probabilidad de ocurrencia $(20 \%)$ pero este va a requerir de acciones inmediatas las cuales pueden desprenderse de dicho escenario.

Luego de la consulta a expertos, se determinó que el escenario apuesta para el sector al año 2025 será el escenario 1 "Jardín del Edén" lo cual originará la formulación de las diversas acciones estratégicas para llegar a él.

\section{CONCLUSIONES}

a) La consecuencia lógica del enfoque actual del sector estudiado es un escenario futurible negativo (Escenario 32 - 0000 "Exterminio total").

b) El factor capacitación según los resultados del análisis es la variable más importante para el sistema en estudio y a la vez es la más inestable (es muy influyente pero a la vez dependiente).

c) Es factible la construcción de un escenario futurible para el sector textil en San Juan de Lurigancho y este debe constituirse como la meta final que guíe las acciones en este sector.

d) Los actores identificados están a favor de los objetivos del sector aunque en la actualidad su accionar no esté totalmente orientado a ellos.

\section{REFERENCIAS BIBLIOGRÁFICAS}

Bendahal, S. (2003). Multi-Issue actor análisis; Journal of Decisión Systems, Vol. 12 № 4.

Cano Alvarán, O. (2004). Metodología para la concertación con actores, 1era. edición, Escuela Superior de Administración Pública, Colombia.

Godet, M. (2000). Caja de herramientas de la prospectiva estratégica, Edición Digital, LIPSOR.

LIPSOR (2005). MICMAC-Se poser les bonnes questions et identifier les variables clés, http:// www.3ie.org/lipsor/micmac.htm

LIPSOR (2005). Analyser les stratégies d'acteurs, http://www.3ie.org/lipsor/mactor.htm

LIPSOR (2005). SMIC-PROB-EXPERT, Impacts croisés probabilistes, http://www.3ie.org/lipsor/ mactor.htm 
López Caicedo, M. (2004). Estudio prospectivo y estratégico Municipio de Fusagasua 2020, Universidad Externado de Colombia, Colombia.

MIDEPLAN (2003). Análisis prospectivo de la provincia de Valdivia para el año 2020, MIDEPLAN, Chile.
Mojica, F.J. (2003). Informe del análisis prospectivo del desarrollo de la región Bogotá-Cundinamarca, Universidad Externado de Colombia, Colombia.

Ospina Toro, G. (2004). Comercialización del gas natural - Plan Estratégico Prospectivo 2004 2015, Universidad Externado de Colombia, Colombia. 